\title{
Modeling and Real-Time Simulation of a Vascularized Liver Tissue
}

\author{
Igor Peterlík, Christian Duriez, and Stéphane Cotin
}

Inria

\begin{abstract}
In Europe only, about 100,000 deaths per year are related to cirrhosis or liver cancer. While surgery remains the option that offers the foremost success rate against such pathologies, several limitations still hinder its widespread development. Among the limiting factors is the lack of accurate planning systems, which has been a motivation for several recent works, aiming at better resection planning and training systems, relying on pre-operative imaging, anatomical and biomechanical modelling. While the vascular network in the liver plays a key role in defining the operative strategy, its influence at a biomechanical level has not been taken into account.

In the paper we propose a real-time model of vascularized organs such as the liver. The model takes into account separate constitutive laws for the parenchyma and vessels, and defines a coupling mechanism between these two entities. In the evaluation section, we present results of in vitro porcine liver experiments that indicate a significant influence of vascular structures on the mechanical behaviour of tissue. We confirm the values obtained in the experiments by computer simulation using standard FEM. Finally, we show that the conventional modelling approach can be efficiently approximated with the proposed composite model capable of real-time calculations.
\end{abstract}

\section{Introduction}

The liver is one of the major organs in the human body and is in charge of more than a hundred vital functions. Because of its many functions, its pathologies are varied, numerous and often lethal. The most advanced state of evolution of these pathologies is cirrhosis or cancer, with nearly 100,000 related deaths in 2008. Surgical procedures remain the option that offers the best success rates against such pathologies, with a 5-year survival rate above $50 \%$ for surgery. Yet, surgery is not always performed due to several limitations, in particular the preoperative estimation of the liver volume remaining after resection. This volume depends highly on the choice of the operative strategy as well as anatomical constraints defined by the vascular network.

Such limitations could be overcome by improving the quality of the planning, which relies on a combination of components, mostly image processing and biomechanical modelling. Several works have addressed this problem. A tool for liver resection planning and training is described in [1] where a model of the liver including the vascular system is reconstructed from CT data and visualized in a 
virtual environment. In [2], a resection map is developed to enhance the resection accuracy by visualizing the structures near to the resection plane. mainly the tumour boundaries and main veins. In [3], techniques for geometrical and structural analysis of vessels are presented. Besides the vessel segmentation and skeletonization based on graph algorithms, the liver parenchyma is segmented into non-overlapping regions according to the supply areas which are visualized during the resection planning. A real-time vascular visualization method is developed in [4. The vessels are modelled as tubular structures and the complete vascular model is projected onto the patient's liver during surgery.

While an important body of work exists regarding the biomechanical properties of the liver parenchyma, only few studies focus on the role of vascularization inside the tissue. In [5], visco-elastic model of the liver is proposed as well as material parameters experimentally measured ex vivo on perfused liver. In [6], a patient specific model of hepatic vasculature is proposed. The material properties of vessels are modelled by non-linear constitutive law. Nevertheless, the model does not allow for real-time performance as the vessel walls are modelled with large number of finite elements.

In this paper a real-time composite model of vascularized organs such as the liver is proposed. The model takes into account separate constitutive laws for the parenchyma and vessels, and defines a coupling mechanism between the two components. As an illustration of mechanical influence of vascularization inside a tissue sample, results conducted on vascularized and non-vascularized tissue samples in vitro are reported. The measured values are further confirmed by a simulation using dense meshes needed for correct discretization of the thin vessel wall. Finally, it is shown that the proposed composite model provides a very good approximation of the conventional model with dense mesh, at the same time being sufficiently fast to run in real-time even for complex geometry.

\section{Methods}

\subsection{Parenchyma Model}

While most studies concerning the material properties of liver parenchyma agree on a viscoelastic behaviour (see [5] or [7] for instance), we employ a simpler nonlinear elastic model. This is essentially motivated by the fact that we are not focusing on the transient part of the deformation but rather the static equilibrium under some specific loading conditions.

The parenchyma is modeled using a finite element co-rotational method. This allows for large displacements or rotations in the model, while relying on a linear expression of the stress-strain relationship. Different methods exist for computing the local rotation of each element; in this paper we use a geometric approach proposed in [8]. In a co-rotational model the stiffness matrix $\mathbf{K}$ depends on the deformation $\mathbf{u}$ and the equation relating the external forces to the displacements can be written as $\mathbf{f}=\mathbf{K}(\mathbf{u}) \mathbf{u}$. The system of equations is solved by direct $\mathrm{LU}$ solver. 

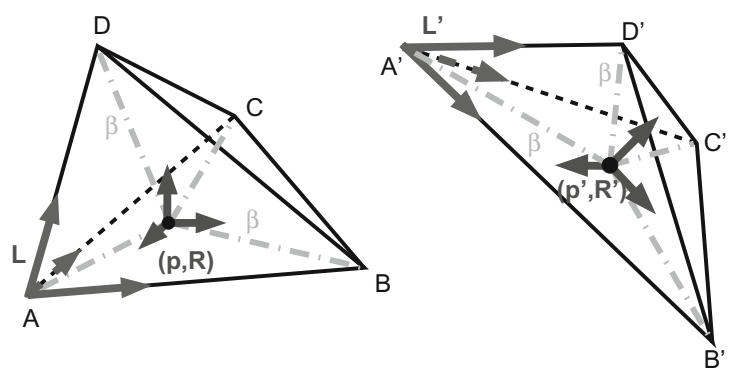

Fig. 1. Mapping between $6 \mathrm{DoF}$ beam node and tetrahedron in initial and rotated positions. The positions of beam and tetrahedron nodes do not coincide.

\subsection{Vessel Model}

As a first step towards a mechanical model of vessels, a continuous representation of vascular structures is constructed. Using segmented data of a vessel tree, points along centerlines of each vessel branch are selected. This task can be performed either manually or automatically, e.g. with VMTK1. Each branch can be represented as a series of cubic Bezier curves fitted to the centerline points: the series begins in a starting point of the branch and ends either in another branching point or in the endpoint of the branch.

A set of interpolated points is constructed using the Bezier curves; positions of the interpolated points can be chosen arbitrarily along each branch dividing it into intervals with a constant or varying length. For each interpolated point we calculate its orientation given by the tangent and normal of the Bezier curve in that point.

The interpolated points are used as nodes of serially linked beam elements, in a similar way as proposed by Duriez et al. 9. for simulating catheters and guidewires. This model shares some similarities with the co-rotational model described above, and in particular allows for geometrically non-linear deformations. We introduce some modifications to the model to take into account the particular nature of vessels, in particular through specific cross section profiles and moments of inertia (see [10] for details). The static formulation for the deformation of a beam is described by a system similar to the one used for the paranchyme, with the difference that each node is described with six degrees of freedom, three of which correspond to the spatial position, and three to the angular position of the node in a global reference frame (see Fig 1).

\subsection{Mechanical Coupling between Vessel and Parenchyma}

We propose a mapping between the mesh nodes of the vessels and parenchyma to create the vascularized model. Since no relative motion between the vessels and parenchyma is observed in reality, the mapping between the two can be modeled as a constraint. In each step of the simulation, the actual displacements

1 wWw.vmtk.org 
of the parenchyma mesh nodes are mapped to the vessel nodes and reciprocally, the force contribution due to the deformation of the vessel is propagated to the parenchyma. The mapping of forces is based on a principle of virtual work. Using the corotational model, the parenchyma is discretized by a mesh composed of linear $P_{1}$ tetrahedral elements given by four nodes with three degrees of freedom. The vessel is modeled with beam elements where each beam is given by two end points each having 6 degrees of freedom (positions and rotations). We focus on mapping between an arbitrary beam node $b$ and tetrahedron $T$.

Let us denote $\mathbf{p}$ the initial position and $\mathbf{R}$ initial rotation of the beam node $b$. Before the simulation starts, we select the tetrahedron $T=\left\{\mathbf{t}_{A}, \mathbf{t}_{B}, \mathbf{t}_{C}, \mathbf{t}_{D}\right\}$ which is closest to the beam node $b$. Then, the barycentric coordinates $\boldsymbol{\beta}_{T}=$ $\left\{\beta_{A}, \beta_{B}, \beta_{C}\right\}$ of the position $\mathbf{p}$ are computed w.r.t. the tetrahedron $T$ and stored. Similarly, the initial rotation $\mathbf{R}$ is transformed to a matrix $\mathbf{O}_{T}$ which defines the orientation of the point $b$ w.r.t. the local basis $\mathbf{L}$ defined by the edges of the tetrahedron, i. e. $\mathbf{O}_{T}=\mathbf{L}^{-1} \mathbf{R}$ (see Fig. 1). According to the initial assumption (no relative motion between parenchyma and vessel), both the barycentric coordinates $\boldsymbol{\beta}_{T}$ and orientation $\mathbf{O}_{T}$ are constant during the simulation. Let's suppose that in the actual step, the tetrahedron is rotated and deformed as shown in Fig. 1. Denoting the nodal positions of the deformed tetrahedron $T^{\prime}=\left\{\mathbf{t}_{A}^{\prime}, \mathbf{t}_{B}^{\prime}, \mathbf{t}_{C}^{\prime}, \mathbf{t}_{D}^{\prime}\right\}$, new actual position $\mathbf{p}^{\prime}$ of the beam node is computed using the barycentric coordinates $\boldsymbol{\beta}_{T}$ as

$$
\mathbf{p}^{\prime}=\mathbf{t}_{A}^{\prime} \beta_{A}+\mathbf{t}_{B}^{\prime} \beta_{B}+\mathbf{t}_{C}^{\prime} \beta_{C}+\mathbf{t}_{D}^{\prime}\left(1-\beta_{A}-\beta_{B}-\beta_{C}\right) .
$$

The updated orientation $\mathbf{R}^{\prime}$ of the beam node is computed using the basis $\mathbf{L}^{\prime}$ given by the actual displacement of the tetrahedron nodes and pre-computed orientation $\mathbf{O}_{T}$. First, $\tilde{\mathbf{R}}^{\prime}=\mathbf{L}^{\prime} \mathbf{O}_{T}$ is computed. However, since besides the rotation a deformation of the tetrahedron must be taken into account, the matrix $\tilde{\mathbf{R}}$ is not necessarily orthogonal. Therefore, the updated orientation $\mathbf{R}^{\prime}$ is calculated as orthogonal component of polar decomposition of $\tilde{\mathbf{R}}^{\prime}$. As the positional mapping is applied in each step of the simulation, the positions of the beams are kinematically linked to the positions of tetrahedra.

After the updated position is mapped from the tetrahedron to the beam point, the forces acting in the beam point must be mapped onto the vertices of the associated tetrahedron. For the beam point, we have linear forces $\mathbf{f}=\left\{f_{x}, f_{y}, f_{z}\right\}$ as well as torques $\boldsymbol{\tau}=\left\{\tau_{x}, \tau_{y}, \tau_{z}\right\}$, whereas for the tetrahedron, there are only linear nodal forces $\mathbf{F}=\left\{\mathbf{F}_{A}, \mathbf{F}_{B}, \mathbf{F}_{C}, \mathbf{F}_{D}\right\}$. The force contribution to each node given by linear forces $\mathbf{f}$ is computed using the barycentric coordinates, required by the positional mapping. The torques are transformed to linear forces acting in the tetrahedron nodes using the equation $\tau=\mathbf{r} \times \mathbf{F}$ where $\mathbf{r}$ is vector connecting the beam and tetrahedron nodes. Putting it together, the force in tetrahedron node $I \in\{A, B, C, D\}$ is computed as $\mathbf{F}_{I}=\beta_{I} \mathbf{f}-\left(\mathbf{t}_{I}^{\prime}-\mathbf{p}^{\prime}\right) \times \boldsymbol{\tau}$. 


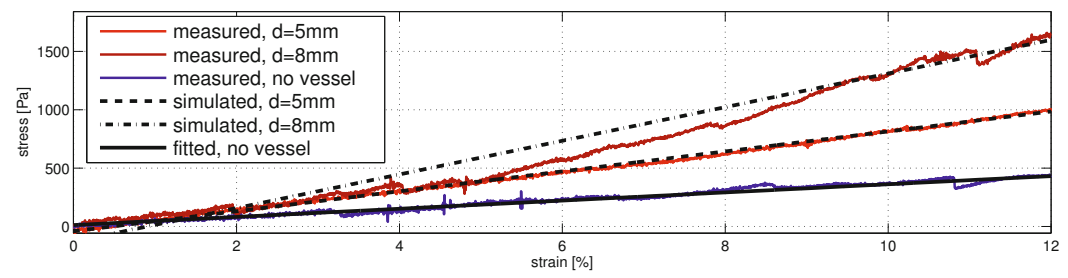

Fig. 2. Experimental and fitted stress-strain curves for three selected samples

\section{Results}

\subsection{In Vitro Measurements of Vascularized Sample Response}

While many studies on liver tissue have been conducted in the past, almost none focused on the evaluation of mechanical properties of hepatic veins. Recently, tensile tests on porcine hepatic veins were performed and reported in [11. The stiffness of vessel wall was experimentally measured and compared to parameters known for other vascular structures in the human body. Nevertheless, to our best knowledge, there has been no experiments evaluating the influence of vessel walls on mechanical behaviour and elastic response of the soft tissue.

To address this gap, we conducted a series of tensile tests on fresh porcine liver using a Bose ElectroForce 3330 Test Instrument. The liver was cut into cube-shaped samples of size approximately $9 \mathrm{~cm}^{3}$. Two main types of samples were extracted: homogeneous and heterogeneous samples with single straight vessel of diameter $d>4 \mathrm{~mm}$. Using cyanoacrylate, the samples were glued on top and bottom face to small wooden plates attached to the test device. The orientation of the vessel was aligned with the direction of the tension.

The top face of the sample was displaced up to $3.5 \mathrm{~mm}$ (about $12 \%$ strain) and the speed of motion was restricted to $1 \mathrm{~mm}$ per minute to emulate quasistatic conditions (the experimental setup is shown in Fig. 3a). During loading, either tissue damage or glue failure occurred in many samples, decreasing the number of reliable measurements. Three representatives were chosen so that during the experiment, none of the issues mentioned above were observed: one homogeneous sample and two vascularized samples having vessels segments with diameters $5 \mathrm{~mm}$ and $8 \mathrm{~mm}$. As mechanical properties of vessel wall was measured, the vessel segments were filled with air. As the gravitational load applied due to the mass of each sample is not negligible w.r.t. the forces being measured, zerostrain state was approximately determined for each tissue and recorded forcedisplacement data were converted into stress - strain curves. For each sample, the stiffness coefficient was obtained using linear fitting: for the homogeneous tissue, the resulting value is an estimation of Young's modulus of the paranchyme $(3.5 \mathrm{kPa})$, whereas for the vascularized samples, the values represent a measure of an apparent stiffness $(8.54 \mathrm{kPa}, 14.41 \mathrm{kPa})$. The measured data (depicted in Fig. (2) indicates that the presence of the vein inside the sample affects significantly the force response of the tissue and the increase in apparent stiffness is proportional to the size of the vessel. 


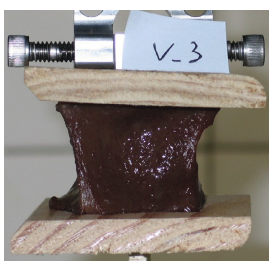

(a)

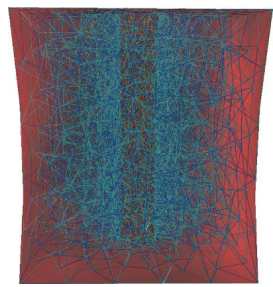

(b)

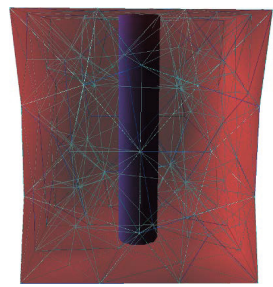

(c)

Fig. 3. Vascularized sample tensile test: (a) Experimental setup, (b) standard FEM with graded mesh, (c) composite model with beams

\subsection{Numerical Simulation of Vascularized Tissue}

In the next step, we reproduced the experiments described above by a numerical simulation in SOFA 2 where both the parenchyma and the vessel wall were discretized with tetrahedral mesh and deformed using standard FEM. Three parameters were needed to set up the model: stiffness of the paranchyme (known from the experiment with homogeneous sample), stiffness and thickness of the vessel wall. As only the apparent stiffness of vascularized samples was known, it was not possible to determine the two unknown parameters uniquely. In [12] the average thickness of human veins is reported to be $150-$ $250 \mu \mathrm{m}$ depending on the diameter of the vein. We use the value $250 \mu \mathrm{m}$ as we are interested mainly in large veins with diameter of $5 \mathrm{~mm}$ and more.

We generated tetrahedral mesh of the vascularized samples; to discretize the thin vessel wall correctly, graded meshes having 37,000 and 52,000 elements (samples with smaller and larger vessel, respectively) were generated (see the mesh depicted in Fig. 3b). The last parameter, vessel wall stiffness, was determined by the simulation: we performed a series of computations for different values of the wall stiffness. Finally, we chose the one that resulted in response force closest to the values measured in vitro. The obtained values of the wall stiffness were determined as 1.1 MPa and 1.4 MPa for the sample with smaller and large vessel, respectively. The Young's modulus of hepatic vein wall reported in [1] is $0.62 \pm 0.41 \mathrm{MPa}$, so our values are slightly higher, but still in the range reported by other studies.

\subsection{Composite Model Evaluation}

The conventional FEM used above requires a huge number of elements even to model a small sample with simple vascularization. We show that a good approximation of the conventional FEM requiring detailed meshes can be achieved by the composite model that we have implemented in SOFA according to section 2 , keeping the number of elements much lower and allowing for real-time calculations. First, in order to perform a numerical validation of the composite model,

${ }^{2}$ www.sofa-framework.org 


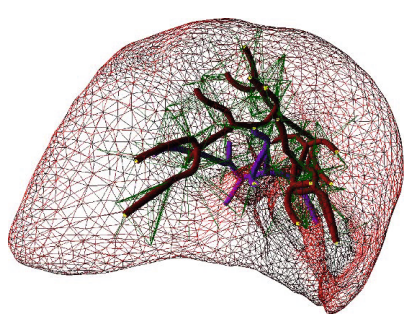

(a)

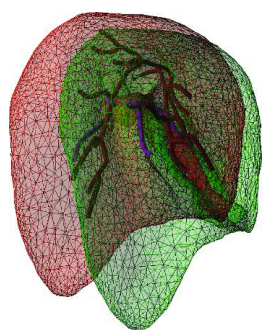

(b)

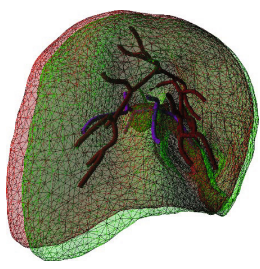

(c)

Fig. 4. Vascularized model of the liver: (a) initial position with mapped vascular trees; (b) comparison of deformation under gravitational loading in vascularized liver with $E_{1}=3.5 \mathrm{kPa}$ (red) and non-vascularized liver (green); (c) comparison of vascularized liver with $E_{2}=15 \mathrm{kPa}$ (red) and non-vascularized liver (green)

we performed the same type of simulation as above, however, we used a homogeneous corotational FEM to model the parenchyma and mapped beam FEM to model the mechanical contribution of the vein. In all computations, the vessel was modelled with two beams coupled to the parenchyma. First, the parenchyma was discretized by a mesh having 1160 elements: the relative errors between the force responses computed by standard and composite FEM was $6.5 \%$ and $2.45 \%$ for smaller and larger vein, respectively. Second, mesh having 170 elements was used for parenchyma, resulting in relative error in force responses of $9 \%$ and $4.6 \%$ for smaller and larger veins. The accuracy of the composite model is emphasized by the fact that the error was significantly lower for samples with large vascularization that has more important influence on the overall elastic response.

In order to demonstrate the efficiency of the algorithm, we performed a simulation of the entire liver: the parenchyma was discretized into 2620 tetrahedra and two vascular trees were modelled inside the liver: hepatic veins composed of 257 beams and hepatic portal veins modelled with 57 beams (Fig $4 \mathrm{~h}$ ). The tetrahedral mesh was fixed on the surface close to the entrance of the hepatic vein. Gravitational loading was applied on the liver, resulting in large displacements.

Two comparisons of vascularized liver (in red) and non-vascularized liver (in green) are presented in Fig团 for two different values of Young's modulus of the parenchyma: $E_{1}=3.5 \mathrm{kPa}$ (Fig,4 $)$ and $E_{2}=15 \mathrm{kPa}$ (Fig 4 4 ). The influence of vascularization is more significant in liver with lower stiffness (difference of more than $5 \mathrm{~cm}$ in deformation between vascularized and non-vascularized model), nevertheless in case of $E_{2}$ the difference in deformation remains over $1 \mathrm{~cm}$ for some parts of the liver. In all experiments, refresh rate of 60 FPS was achieved on PC with CPU Intel CPU i7-2630QM running at $2.00 \mathrm{GHz}$.

\section{Conclusion}

In the paper we focused on mechanical modeling of vascularized tissues. We introduced a novel approach based on modelling the parenchyma and vessels as 
two separated entities, mechanically coupled with a mapping of position and forces. The experiments presented in the evaluation section confirmed that vascular structures play important role in tissue behaviour. We also evaluated the composite model, comparing the relative error w.r.t. standard detailed FEM. Finally, we demonstrated that proposed model can be used in real-time even for complicated structures, such as liver organ with two vascular trees.

In future work, we plan to employ the composite model in inverse problems that appear in elastography. Also, we want to propose similar approach for mapping between parenchyma and thin two-dimensional structures to model Glisson capsule on the surface of the liver.

\section{References}

1. Sojar, V., Stanisavljevic, D., Hribernik, M., Glusic, M., Kreuh, D., Velkavrh, U., Fius, T.: Liver surgery training and planning in $3 \mathrm{~d}$ virtual space. International Congress Series, vol. 1268, pp. 390-394 (2004)

2. Lamata, P., Jalote-Parmar, A., Lamata, F., Declerck, J.: The resection map, a proposal for intraoperative hepatectomy guidance. International Journal of Computer Assisted Radiology and Surgery 3(3-4), 299-306 (2008)

3. Selle, D., Preim, B., Schenk, A., Peitgen, H.: Analysis of vasculature for liver surgical planning. IEEE Trans. on Medical Imaging 21(11), 1344-1357 (2002)

4. Ritter, F., Hansen, C., Dicken, V., Konrad, O., Preim, B., Peitgen, H.O.: Realtime illustration of vascular structures. IEEE Trans. on Visual. and Computer Graphics 12, 877-884 (2006)

5. Kerdok, A.E., Ottensmeyer, M.P., Howe, R.D.: Effects of perfusion on the viscoelastic characteristics of liver. Journal of Biomechanics 39, 2221-2231 (2006)

6. Nguyen, B.P., Yang, T., Leon, F., Chang, S., Ong, S.H., Chui, C.K.: Patient Specific Biomechanical Modeling of Hepatic Vasculature for Augmented Reality Surgery. In: 4th International Workshop on Medical Imaging and Augmented Reality (2008)

7. Gao, Z., Kim, T., James, D.L., Desai, J.P.: Semi-automated soft-tissue acquisition and modeling forsurgical simulation. In: CASE 2009: Proceedings of the Fifth Annual IEEE International Conference on Automation Science and Engineering, Piscataway, NJ, USA, pp. 268-273. IEEE Press (2009)

8. Nesme, M., Payan, Y., Faure, F.: Efficient, physically plausible finite elements. In: Dingliana, J., Ganovelli, F. (eds.) Eurographics 2005, Short papers, Trinity College, Dublin, Irlande (August 2005)

9. Duriez, C., Cotin, S., Lenoir, J., Neumann, P.F.: New approaches to catheter navigation for interventional radiology simulation. Computer Aided Surgery 11, 300-308 (2006)

10. Przemieniecki, J.S.: Theory of Matrix Structural Analysis (1985); reprint of McGraw Hill (1968)

11. Umale, S., Chatelin, S., Bourdet, N., Deck, C., Diana, M., Dhumane, P., Soler, L., Marescaux, J., Willinger, R.: Experimental in vitro mechanical characterization of porcine glisson's capsule and hepatic veins. J. of Biomech. 44, 1678-1683 (2011)

12. Forauer, A., Theoharis, C.: Histologic changes in the human vein wall adjacent to indwelling central venous catheters. J. of Vasc. and Interv. Rad. 14, 1163-1168 (2003) 